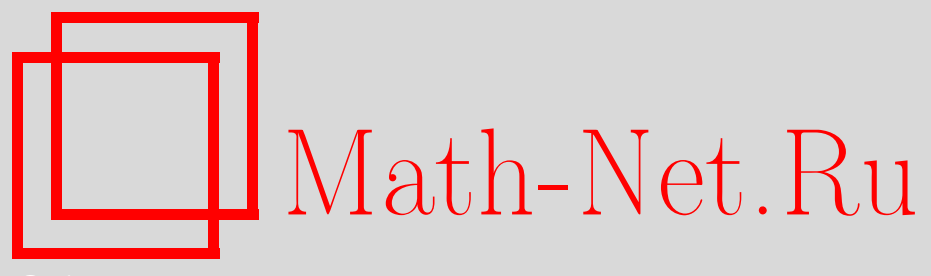

Д. В. Христофоров, О явлении ложной интерполяции эллиптических функций диагональными аппроксимациями Паде, Матем. заметки, 2010, том 87, выпуск 4, 604615

DOI: https://doi.org/10.4213/mzm7708

Использование Общероссийского математического портала Math-Net.Ru подразумевает, что вы прочитали и согласны с пользовательским соглашением http://www . mathnet.ru/rus/agreement

Параметры загрузки:

IP: 35.174 .16 .151

26 апреля 2023 г., 12:30:20

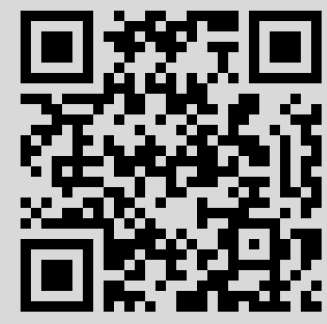


Том 87 выпуск 4 апрель 2010

УДК 517.53

\section{О явлении ложной интерполяции эллиптических функций диагональными аппроксимациями Паде}

\section{Д. В. Христофоров}

В работе изучаются диагональные аппроксимации Паде для эллиптических функций. Наличие ложных полюсов аппроксимаций, не соответствующих особенностям приближаемой функции, препятствует их равномерной сходимости в области Шталя. Это явление оказывается тесно связанным с существованием в области Шталя точек ложной интерполяции, в которых аппроксимации Паде интерполируют другую ветвь эллиптической функции. В работе исследуется поведение диагональных аппроксимаций Паде в окрестности точек ложной интерполяции.

Библиография: 14 названий.

\section{1. Введение}

Пусть функция $f$ задана сходящимся рядом Лорана в окрестности точки $z=\infty$ :

$$
f(z)=\sum_{k=0}^{\infty} \frac{c_{k}}{z^{k}}
$$

Рассмотрим последовательность аппроксимаций Паде $f_{n}(z):=p_{n}(z) / q_{n}(z)$, где полиномы $p_{n}$ и $q_{n}$ имеют степень не выше $n, q_{n} \not \equiv 0$, и удовлетворяют условию касания

$$
R_{n}(z):=q_{n}(z) f(z)-p_{n}(z)=O\left(\frac{1}{z^{n+1}}\right), \quad z \rightarrow \infty .
$$

Если $\operatorname{deg} q_{n}=n, \operatorname{deg} p_{n}=n$ и полиномы $p_{n}$ и $q_{n}$ взаимно просты, то индекс $n$ называется нормальным.

Согласно общим результатам Шталя [1] если ряд (1.1) представляет алгебраическую функцию, то существует “симметричный” компакт (в дальнейшем - компакт Шталя) $S=S(f) \subset \mathbb{C}$ со следующими свойствами:

(1) $S$ состоит из конечного числа кусочно-аналитических дуг и множество $D:=\overline{\mathbb{C}} \backslash S-$ область;

(2) $f$ продолжается мероморфным образом в область $D=D(f)$;

Работа выполнена при поддержке Российского фонда фундаментальных исследований (грант № 08-01-00317) и программы "Ведущие научные школы" (грант № НШ-3906.2008.1).

(C) Д. В. Христофоров, 2010 
(3) $f_{n} \stackrel{\text { сар }}{\longrightarrow} f$ на компактных подмножествах области $D$, где $f_{n}$ - диагональнъе аппроксимации Паде для функиии $f$ в точке $z=\infty$, а $\stackrel{\text { сар }}{\longrightarrow}$ означает сходимость по емкости.

Область Шталя $D=\overline{\mathbb{C}} \backslash S$ является максимальной областью сходимости по емкости последовательности $\left\{f_{n}\right\}$. Основным препятствием (см. [2]-[6]) к равномерной сходимости аппроксимаций Паде является наличие ложных полюсов - полюсов рациональных функций $f_{n}$, имеющих предельные точки, компактно принадлежащие области $D$ и не совпадающие с особенностями функции $f$ в $D$. Из теоремы Шталя о сходимости по емкости следует, что асимптотически ложные полюсы совпадают с нулями функций $f_{n}$, такие пары называются дефектами [7]. Часто полагают [7]-[9], что дефекты аппроксимаций Паде являются случайными и никак не связаны с природой приближаемой функции.

В 1908 г. Дюма [10] исследовал сходимость чебышевской непрерывной дроби для эллиптической функции вида

$$
f(z)=w(z)-z^{2}+\frac{e_{1}+e_{2}+e_{3}+e_{4}}{2} z,
$$

где $w(z)=\sqrt{\left(z-e_{1}\right)\left(z-e_{2}\right)\left(z-e_{3}\right)\left(z-e_{4}\right)}, e_{1}, \ldots, e_{4}-$ попарно различные точки на комплексной плоскости и выбранная ветвь корня обеспечивает голоморфность функции $f$ в бесконечности. Дюма показал, что в "общем положении" для некоторой бесконечной подпоследовательности $\Lambda \subset \mathbb{N}$ у диагональных аппроксимаций $f_{n}$, $n \in \Lambda$, существуют полюсы, множество которых всюду плотно в $\overline{\mathbb{C}}$, а динамика в зависимости от $n$ подчиняется определенной закономерности.

Результат Дюма был перенесен Суетиным [4] на класс функиий, включающий в себя эллиптические функции (1.3). Было отмечено следующее явление, тесно связанное с наличием ложных полюсов: для каждого $n$ из некоторой бесконечной подпоследовательности $\Lambda \subset \mathbb{N}$ существует точка $\beta_{n} \in D$, для которой $f_{n}\left(\beta_{n}\right)=$ $\widetilde{f}\left(\beta_{n}\right)$, где функция $\widetilde{f}-$ другая ветвь функции $f$ :

$$
\widetilde{f}(z)=-w(z)-z^{2}+\frac{e_{1}+e_{2}+e_{3}+e_{4}}{2} z .
$$

Это означает, что аппроксимации Паде интерполируют в некоторых точках области Шталя $D$ другую ветвъ функции $f$. На этом явлении основан, например, контрпример Буслаева [11] к гипотезе Бейкера-Грейвс-Морриса. В случае рациональной независимости неких вещественных параметров $\tau_{1}=\tau_{1}\left(e_{1}, e_{2}, e_{3}, e_{4}\right), \tau_{2}=\tau_{2}\left(e_{1}, e_{2}, e_{3}, e_{4}\right)$ и единицы (см. [4]) $\left\{\beta_{n}, n \in \Lambda\right\}^{\prime}=\overline{\mathbb{C}}$.

Тем самым, в области Шталя последовательность $\left\{f_{n}\right\}$ не может равномерно сходиться к функции $f(1.3)$. Отметим, что задача о равномерном приближении функции $f$ диагональными аппроксимациями Паде в области Шталя рассматривается в [12].

Пусть функция $f$, заданная рядом (1.1), имеет вид

$$
f(z)=\frac{w(z)}{q(z)}-r(z)
$$

где $w(z)$ из (1.3), $q \in \mathbb{C}[z]$ - полином фиксированной степени $m$, все нули которого расположены в области Шталя, $r \in \mathbb{C}(z)$ есть сумма главных частей мероморфной в области $D$ функции $w(z) / q(z)$. Таким образом, функция $f \in \mathscr{H}(D)$. 
Пусть $\rho(x, y)$ - расстояние в сферической метрике в $\overline{\mathbb{C}}$ между точками $x$ и $y$. Тогда имеет место следующая

Теорема. Пусть точки $e_{1}, \ldots, e_{4}$ таковљ, что компакт Шталя $S$ для функи,и

$$
w(z)=\sqrt{\left(z-e_{1}\right)\left(z-e_{2}\right)\left(z-e_{3}\right)\left(z-e_{4}\right)}
$$

состоит из двух непересекающихся дуг $S_{1}$ и $S_{2}$ и заданные вещественные параметры $\tau_{1}\left(e_{1}, \ldots, e_{4}\right), \tau_{2}\left(e_{1}, \ldots, e_{4}\right)$ и единица рационально независимы. Предположим, что для эллиптической функции $f(z)=w(z) / q(z)-r(z)$, голоморфной в области $D=\overline{\mathbb{C}} \backslash S$, все достаточно большие индексы $n$ нормальны, m.e. ord $f_{n}=n$. Тогда существует последовательность $\widetilde{\Lambda}=\widetilde{\Lambda}(f)$ натуральных чисел, точки $\beta_{n} \in \mathbb{C} \backslash S$, $n \in \widetilde{\Lambda}, u$ числа $\rho_{n} \downarrow 0, n \in \widetilde{\Lambda}$, такие, что

$$
f_{n}\left(\beta_{n}\right)=\tilde{f}\left(\beta_{n}\right) \quad u \quad f_{n}\left(K_{n}^{\rho_{n}}\right)=\overline{\mathbb{C}}, \quad \text { ¿de } \quad K_{n}^{\rho_{n}}=\left\{z \in D: \rho\left(z, \beta_{n}\right)<\rho_{n}\right\} .
$$

Условия теоремы определяют случай “общего положения”, в предположении которого мы будем проводить доказательство в этой работе.

Зафиксируем произвольное $\varepsilon>0$ и обозначим через $\Lambda^{\varepsilon}$ множество $n \in \Lambda$ таких, что $\beta_{n} \in D^{\varepsilon}:=\overline{\mathbb{C}} \backslash S^{\varepsilon}, S^{\varepsilon}-2 \varepsilon$-окрестность компакта $S$. В качестве последовательности $\widetilde{\Lambda}$ в теореме мы будем брать последовательность $\Lambda^{\varepsilon}$.

Данная теорема является уточнением сформулировнной в [6] теоремы, в которой радиус $\rho_{n}$ окрестностей $K_{n}^{\rho_{n}}$ фиксирован.

Дальнейшая схема построения работы следующая. В разделе 2 приведены основные понятия и результаты, связанные с теорией римановых поверхностей, которые необходимы при доказательстве теоремы. В разделе 3 выводится явный вид функции остатка $R_{n}$ из (1.2). Наконец, в разделе 4 доказывается некоторая вспомогательная лемма и затем приводится доказательство сформулированной теоремы.

\section{2. Основные понятия и вспомогательные результаты}

2.1. Доказательства будем проводить при помощи стандартных конструкций на эллиптической римановой поверхности $X$, заданной уравнением $w^{2}=\left(z-e_{1}\right) \times$ $\left(z-e_{2}\right)\left(z-e_{3}\right)\left(z-e_{4}\right)$. Реализуем $X$ как два экземпляра $\overline{\mathbb{C}}$, разрезанных по дугам $S_{1}$ и $S_{2}$ компакта Шталя. На каждой из дуг зададим ориентацию: $S_{1}=\operatorname{arc}\left(e_{1}, e_{2}\right)$, положительная ориентация от $e_{1} \mathrm{k} e_{2} ; S_{2}=\operatorname{arc}\left(e_{3}, e_{4}\right)$, положительная ориентация от $e_{3} \mathrm{~K} e_{4}$. Соответствующим образом определяются "верхний" и "нижний" берега разрезов, а два экземпляра расширенной комплексной плоскости оказываются "склеены" накрест. Компакту $S$ при этом на римановой поверхности соответствует пара непересекающихся кривых $\mathscr{L}_{1}$ и $\mathscr{L}_{2}, \mathscr{L}:=\mathscr{L}_{1} \cup \mathscr{L}_{2}$.

Зафиксируем однозначную в области $D=\overline{\mathbb{C}} \backslash S$ ветвь квадратного корня соотношением

$$
\frac{\sqrt{\left(z-e_{1}\right) \cdots\left(z-e_{4}\right)}}{z^{2}} \rightarrow 1, \quad z \rightarrow \infty
$$

Точка $\mathbf{z} \in X$ определяется своей проекцией $z=\pi(\mathbf{z}), z \in \overline{\mathbb{C}}$, и номером листа римановой поверхности. На первом открытом листе $D^{(1)}$ выполняется соотношение $w=\sqrt{\left(z-e_{1}\right)\left(z-e_{2}\right)\left(z-e_{3}\right)\left(z-e_{4}\right)}$, на втором открытом листе $D^{(2)}$ - соотношение 
$w=-\sqrt{\left(z-e_{1}\right)\left(z-e_{2}\right)\left(z-e_{3}\right)\left(z-e_{4}\right)}$. Таким образом, $\mathbf{z}=(z, \pm w)$. $\mathbf{C}$ первым листом мы будем отождествлять область $D$.

Будем использовать обозначение $z^{(1)}$ при $\mathbf{z} \in D^{(1)}$ и $z^{(2)}$ при $\mathbf{z} \in D^{(2)}$.

Зададим базис гомологий на $X$. Первый из контуров $\mathscr{L}_{1}$ выберем в качестве базисного $a$-цикла $\mathbf{a}_{1}$ и дополним его $b$-циклом $\mathbf{b}_{1}, \mathbf{a}_{1} \circ \mathbf{b}_{1}=1$. Пространство голоморфных дифференциалов на $X$ одномерно; обозначим через $d \Omega_{1} a$-нормированный абелев дифференциал. Имеем

$$
\oint_{\mathbf{a}_{1}} d \Omega_{1}(\boldsymbol{\zeta})=1, \quad \oint_{\mathbf{b}_{1}} d \Omega_{1}(\boldsymbol{\zeta})=B_{1},
$$

где $\operatorname{Im} B_{1}>0$.

2.2. Пусть $\mathbf{z}_{1}$ и $\mathbf{z}_{2}$ - произвольные точки римановой поверхности $X$. Обозначим через $d \Omega\left(\mathbf{z}_{1}, \mathbf{z}_{2} ; \mathbf{z}\right)$ абелев дифференциал третьего рода, голоморфный в $X \backslash\left\{\mathbf{z}_{1}, \mathbf{z}_{2}\right\}$ и имеющий простые полюсы в выделенных точках с вычетами 1 и -1 соответственно и нормированный условием $\oint_{\mathbf{a}_{1}} d \Omega\left(\mathbf{z}_{1}, \mathbf{z}_{2} ; \mathbf{z}\right)=0$. Такой дифференциал называется a-нормированным. Приведем здесь явный вид некоторых дифференциалов, который будет необходим при доказательстве:

$$
\begin{aligned}
d \Omega\left(\mathbf{z}_{1}, \mathbf{z}_{2} ; \mathbf{z}\right) & =\frac{1}{2}\left\{\frac{w(\mathbf{z})+w\left(\mathbf{z}_{1}\right)}{z-z_{1}} \frac{d z}{w(\mathbf{z})}-\frac{w(\mathbf{z})+w\left(\mathbf{z}_{2}\right)}{z-z_{2}} \frac{d z}{w(\mathbf{z})}+\frac{c_{1}}{w(\mathbf{z})}\right\}, \\
d \Omega\left(\mathbf{z}_{1}, \infty^{(2)} ; \mathbf{z}\right) & =\frac{1}{2} \frac{w(\mathbf{z})+w\left(\mathbf{z}_{1}\right)}{z-z_{1}} \frac{d z}{w(\mathbf{z})}-\frac{1}{2} \frac{w(\mathbf{z})-c_{2}}{w(\mathbf{z})} d z \\
d \Omega\left(\infty^{(1)}, \infty^{(2)} ; \mathbf{z}\right) & =-\frac{z-c_{3}}{w(\mathbf{z})} d z
\end{aligned}
$$

где константы $c_{j} \in \mathbb{C}$ обеспечивают $a$-нормированность дифференциалов.

В дальнейшем будем пользоваться интегралами от абелевых дифференциалов по путям, лежащим в рассеченной римановой поверхности. Рассеченная риманова поверхность $\tilde{X}$ определяется как $\tilde{X}:=X \backslash\left(\mathbf{a}_{1} \cup \mathbf{b}_{1}\right)$ и является связной. Справедливо следующее соотношение Римана (см. [13], [14]), которым будем пользоваться в дальнейшем:

$$
\oint_{\mathbf{b}_{1}} d \Omega\left(\mathbf{z}_{1}, \mathbf{z}_{2} ; \boldsymbol{\zeta}\right)=-2 \pi i \int_{\mathbf{z}_{1}}^{\mathbf{z}_{2}} d \Omega_{1}(\boldsymbol{\zeta}),
$$

где путь интегрирования от $\mathbf{z}_{1}$ к $\mathbf{z}_{2}$ лежит в $\widetilde{X}$.

Кроме $a$-нормированных дифференциалов в процессе доказательства нам понадобится дифференциал комплексной функции Грина. Пусть $g_{D}(z, \infty)$ - функция Грина области $D$, а $\widetilde{g}_{D}(z, \infty)$ - гармонически сопряженная к ней, $G_{D}(z, \infty)=$ $g_{D}(z, \infty)+i \widetilde{g}_{D}(z, \infty)$ - многозначная комплексная функция Грина. Дифференциал комплексной функции Грина $d G\left(\infty^{(1)}, \infty^{(2)} ; \mathbf{z}\right)$ имеет полюсы с вычетами \pm 1 в обеих бесконечно удаленных точках римановой поверхности и чисто мнимые периоды. Справедливо следующее соотношение [4]:

$$
d G\left(\infty^{(1)}, \infty^{(2)} ; \mathbf{z}\right)+2 \pi i \omega_{1}(\infty) d \Omega_{1}(\mathbf{z})+d \Omega\left(\infty^{(1)}, \infty^{(2)} ; \mathbf{z}\right)=0,
$$

где $\omega_{1}(\infty)$ - гармоническая мера дуги $S_{1}$ относительно области $D$. Равенство $(2.3)$ вытекает непосредственно из соотношения

$$
\omega_{1}(\infty)=-\frac{1}{2 \pi i} \oint_{\mathbf{a}_{1}} d G\left(\infty^{(1)}, \infty^{(2)} ; \mathbf{z}\right) .
$$


В будущем нам понадобится вещественная величина

$$
\tau_{1}(\infty)=\frac{1}{2 \pi i} \oint_{\mathbf{b}_{1}} d G\left(\infty^{(1)}, \infty^{(2)} ; \mathbf{z}\right)
$$

2.3. Дивизором $(F)$ мероморфной на $X$ функции $F$ будем называть формальное выражение

$$
(F)=n_{1} \mathbf{z}_{1}+n_{2} \mathbf{z}_{2}+\cdots+n_{p} \mathbf{z}_{p}-k_{1} \boldsymbol{\zeta}_{1}-k_{2} \boldsymbol{\zeta}_{2}-\cdots-k_{q} \boldsymbol{\zeta}_{q},
$$

где $\mathbf{z}_{j}$ - нули функции $F$, а $n_{j}-$ их кратности, $\boldsymbol{\zeta}_{j}-$ полюсы функции $F, k_{j}-$ их кратности. По теореме Абеля $\operatorname{deg}(F)=0$ и

$$
\sum_{j=1}^{p} n_{j} \Omega_{1}\left(\mathbf{z}_{j}\right)-\sum_{j=1}^{q} k_{j} \Omega_{1}\left(\boldsymbol{\zeta}_{j}\right) \equiv 0, \quad \Omega_{1}(\mathbf{z})=\int_{e_{3}}^{\mathbf{z}} d \Omega_{1}(\boldsymbol{\zeta})
$$

равенство понимается по модулю периодов базисного голоморфного дифференциала $d \Omega_{1}(\mathbf{z})$.

\section{3. Явный вид функции остатка}

3.1. При фиксированном $n$ рассмотрим функцию $R_{n}$ как заданную на римановой поверхности $X$. Исходя из (1.2) и (1.4) имеем

$$
\begin{aligned}
R_{n}\left(z^{(2)}\right) & =q_{n}(z) \widetilde{f}(z)-p_{n}(z)=-q_{n}(z) \frac{w(z)}{q(z)}-q_{n}(z) r(z)-p_{n}(z) \\
& =R_{n}\left(z^{(1)}\right)-2 q_{n}(z) \frac{w(z)}{q(z)} .
\end{aligned}
$$

Отсюда с учетом условия касания (1.2) имеем

$$
R_{n}(\mathbf{z})= \begin{cases}O\left(z^{-(n+1)}\right), & \mathbf{z} \rightarrow \infty^{(1)} \\ O\left(z^{n+2-m}\right), & \mathbf{z} \rightarrow \infty^{(2)} \\ O\left(\left(z-a_{j}^{(2)}\right)^{-k_{j}}\right), & \mathbf{z} \rightarrow a_{j}^{(2)}, j=1, \ldots, m\end{cases}
$$

где $a_{j}^{(2)} \in D^{(2)}$ соответствуют нулям полинома $q(z)$, а $k_{j}$ - их кратностям. Задача о нахождении функции $R_{n}$ переформулируется следующим образом.

ЗАДАчА. При фиксированном $n \in \mathbb{N}$ найти функцию $R_{n}$ такую, что

1) $R_{n}$ мероморфная на $X$;

2) дивизор

$$
\left(R_{n}\right)=\mathbf{z}_{n}+(n+1) \infty^{(1)}-(n+2-m) \infty^{(2)}-a_{1}^{(2)}-\cdots-a_{m}^{(2)} .
$$

Неизвестный нуль $\mathbf{z}_{n}$ появляется из условия $\operatorname{deg}\left(R_{n}\right)=0$. Данными условиями искомая функция $R_{n}$ определяется однозначно с точностью до мультипликативной постоянной. 
3.2. Приведем явное решение задачи. Для этого рассмотрим $a$-нормированные абелевы дифференциалы $d \Omega\left(\mathbf{z}_{1}, \mathbf{z}_{2} ; \mathbf{z}\right)$ и соответствующие им интегралы $\Omega\left(\mathbf{z}_{1}, \mathbf{z}_{2} ; \mathbf{z}\right)=$ $\int_{e_{3}}^{\mathbf{z}} d \Omega\left(\mathbf{z}_{1}, \mathbf{z}_{2} ; \zeta\right)$, где интегрирование ведется по рассеченной римановой поверхности $\widetilde{X}$. Введем функцию

$$
F(z):=\exp \left\{(n+1-m) \Omega\left(\infty^{(1)}, \infty^{(2)} ; \mathbf{z}\right)+\Omega\left(\mathbf{z}_{n}, \infty^{(2)} ; \mathbf{z}\right)+\sum_{j=1}^{m} \Omega\left(\infty^{(1)}, a_{j}^{(2)} ; \mathbf{z}\right)\right\} .
$$

Имеем $(F)=\left(R_{n}\right)$. В силу $а$-нормированности используемых дифференциалов период функции $F$ при обходе а 1 -цикла равен нулю. Подправим функцию $F$ таким образом, чтобы ее период при обходе $\mathbf{b}_{1}$-цикла также равнялся нулю. Тогда новая функция станет мероморфной на поверхности $X$ и, следовательно, совпадет с функцией $R_{n}$.

Из теоремы Абеля следует

$$
(n+1) \Omega_{1}\left(\infty^{(1)}\right)+\Omega_{1}\left(\mathbf{z}_{n}\right)-(n+2-m) \Omega_{1}\left(\infty^{(2)}\right)-\sum_{j=1}^{m} \Omega_{1}\left(a_{j}^{(2)}\right)=0 \quad \bmod \left(1, B_{1}\right),
$$

где $B_{1}-\mathbf{b}_{1}$-период дифференциала $\Omega_{1}, \operatorname{Im} B_{1}>0$. Если правую часть (3.2) представить как линейную комбинацию периодов и использовать интегральное представление $\Omega_{1}(\mathbf{z})=\int_{e_{3}}^{\mathbf{z}} d \Omega(\xi)$, получим

$$
\begin{aligned}
& (n+1) \int_{e_{3}}^{\infty^{(1)}} d \Omega_{1}(\mathbf{z})+\int_{e_{3}}^{\mathbf{z}_{n}} d \Omega_{1}(\mathbf{z})-(n+2-m) \int_{e_{3}}^{\infty^{(2)}} d \Omega_{1}(\mathbf{z}) \\
& \quad-\sum_{j=1}^{m} \int_{e_{3}}^{\mathbf{q}_{j}} d \Omega_{1}(\mathbf{z})-l_{n}-k_{n} B_{1}=0,
\end{aligned}
$$

где $l_{n}, k_{n} \in \mathbb{Z}$. Рассмотрим новую функцию

$$
\begin{aligned}
\widehat{F}(z):=\exp \{(n+ & 1-m) \Omega\left(\infty^{(1)}, \infty^{(2)} ; \mathbf{z}\right)+\Omega\left(\mathbf{z}_{n}, \infty^{(2)} ; \mathbf{z}\right) \\
& \left.+\sum_{j=1}^{m} \Omega\left(\infty^{(1)}, a_{j}^{(2)} ; \mathbf{z}\right)+2 \pi i k_{n} \Omega_{1}(z)\right\} .
\end{aligned}
$$

Для вычисления ее $\mathbf{b}_{1}$-периода $T$ вычислим входящие под знак экспоненты в (3.4) интегралы по $\mathbf{b}_{1}$-циклу и применим соотношение Римана (2.2):

$$
T=-2 \pi i\left\{(n+1-m) \int_{\infty}^{\infty^{(1)}} d \Omega_{1}(\mathbf{z})+\int_{\mathbf{z}_{n}}^{\infty^{(2)}} d \Omega_{1}(\mathbf{z})+\sum_{j=1}^{m} \int_{\infty^{(1)}}^{a_{j}^{(2)}} d \Omega_{1}(\mathbf{z})-k_{n} B_{1}\right\}
$$

Прибавим к (3.5) равенство (3.3), предварительно умножив его на $-2 \pi i$. Получим

$$
T=2 \pi i l_{n}
$$

Отсюда следует, что $\widehat{F}$ - мероморфная функция на римановой поверхности $X$, имеющая требуемый дивизор и, следовательно, $\widehat{F}=R_{n}$ с точностью до мультипликативной константы. 
Используя соотношение (2.3), перепишем (3.4) следующим образом:

$$
\begin{aligned}
\widehat{F}(z):=\exp \{-(n & +1-m) G(\mathbf{z})+\Omega\left(\mathbf{z}_{n}, \infty^{(2)} ; \mathbf{z}\right)+\sum_{j=1}^{m} \Omega\left(\infty^{(1)}, a_{j}^{(2)} ; \mathbf{z}\right) \\
& \left.+2 \pi i k_{n} \Omega_{1}(z)-2 \pi i(n+1-m) \omega_{1}(\infty) \Omega_{1}(\mathbf{z})\right\} .
\end{aligned}
$$

Интегрируя соотношение (2.3) по $\mathbf{b}_{1}$-циклу, получим

$$
\int_{\infty(1)}^{\infty^{(2)}} d \Omega_{1}(\mathbf{z})=\tau_{1}(\infty)+\omega_{1}(\infty) B_{1}
$$

Так как в (3.3) нижний предел интегрирования можно выбрать любой фиксированный, заменим $e_{3}$ на $\infty^{(1)}$ и используем (3.6):

$$
(n+2-m) \tau_{1}(\infty)+(n+2-m) \omega_{1}(\infty) B_{1}-\int_{\infty(1)}^{\mathbf{z}_{n}} d \Omega_{1}(\mathbf{z})+\sum_{j=1}^{m} \int_{\infty(1)}^{\mathbf{q}_{j}} d \Omega_{1}(\mathbf{z})=l_{n}+k_{n} B_{1} .
$$

Взяв мнимую часть этого равенства с учетом $\operatorname{Im} B_{1}>0$, получаем представление для числа $k_{n}$ :

$$
k_{n}=(n+1-m) \omega_{1}(\infty)+\gamma_{n}, \quad \gamma_{n} \in \mathbb{R} .
$$

Заметим, что величина $\gamma_{n}$, возникающая в (3.8), равномерно ограничена по $n: \gamma_{n}=$ $O(1), n \rightarrow \infty$. Для доказательства этого факта достаточно воспользоваться точным равенством (3.7), рассмотрев его мнимую часть. Так как интегрирование идет по рассеченной римановой поверхности $\widetilde{X}$, то величина $\operatorname{Im} \int_{\infty(1)}^{\mathbf{z}_{n}} d \Omega_{1}(\mathbf{z})$ равномерно ограничена при $\mathbf{z}_{n} \in X$. Следовательно, будет равномерно ограничена по $n$ и величина $\gamma_{n}$.

Окончательно получаем

$$
R_{n}(\mathbf{z})=h_{n} \Phi^{(n+1-m)}(\mathbf{z}) \exp \left\{\Omega\left(\mathbf{z}_{n}, \infty^{(2)} ; \mathbf{z}\right)+\sum_{j=1}^{m} \Omega\left(\infty^{(1)}, a_{j}^{(2)} ; \mathbf{z}\right)\right\} \exp \left\{2 \pi i \gamma_{n} \Omega_{1}(\mathbf{z})\right\}
$$

где $h_{n} \neq 0$ - мультипликативная постоянная.

\section{4. Доказательство теоремы}

4.1. Пусть $\rho(x, y)$ - сферическая метрика в $\overline{\mathbb{C}}$ :

$$
\rho(x, y)=\frac{|x-y|}{\sqrt{1+|x|^{2}} \sqrt{1+|y|^{2}}} .
$$

Докажем следующее утверждение, которое потребуется в дальнейшем.

ЛЕмма. Пусть $f$-алгебраическая функиия, заданная и однозначная на компакте $E$ в расширенной комплексной плоскости $\overline{\mathbb{C}}$, а $K$ - некоторый компакт в комплексной плоскости $\mathbb{C}$. Тогда для любого $a \in K$ и для любого $\varepsilon>0$ существует конечное оббединение кругов $E_{a}(\varepsilon):=\bigcup_{k=1}^{s(a)} B_{k, r(\varepsilon)}$ с иентрами на компакте $E$, имеющих в сферической метрике радиус $r(\varepsilon)=r(\varepsilon, a)$, такое, что 
(1) $\{z:|f(z)-a|<\varepsilon, z \in E\} \subseteq E_{a}(\varepsilon)$;

(2) $r(\varepsilon, a) \rightrightarrows 0$ при $\varepsilon \rightarrow 0$ и равномерно по $a \in K$.

При этом число кругов $s(a)$ равномерно ограничено по $a \in K$.

В силу алгебраичности функции $f$ для любого $a \in K$ множество

$$
A:=\{z: f(z)-a=0, z \in E\}
$$

состоит из не более чем конечного числа $s$ точек. Это множество для каждого фиксированного $a$ возьмем в качестве центров кругов из утверждения леммы, а радиус $r(\varepsilon, a)$ определим как

$$
r(\varepsilon, a):=\sup _{z:|f(z)-a|<\varepsilon} \rho(z, A) .
$$

Отсюда вытекает утверждение (1) и выполняется равномерная оценка $s(a) \leqslant s$.

Фиксируем $a \in K$ и покажем, что $r(\varepsilon, a) \rightarrow 0$ при $\varepsilon \rightarrow 0$. Предположим противное. Тогда существуют последовательности $\left\{z_{k}\right\}_{k=1}^{\infty} \subset K,\left\{\varepsilon_{k}\right\}_{k=1}^{\infty} \subset \mathbb{R}$ и число $c>0$ такие, что

$$
\rho\left(z_{k}, A\right)>c \quad \text { и } \quad\left|f\left(z_{k}\right)-a\right|<\varepsilon_{k}, \quad \varepsilon_{k} \rightarrow 0, \quad k \rightarrow \infty .
$$

Так как $E$ - компакт в $\overline{\mathbb{C}}$, мы можем выбрать сходящуюся подпоследовательность $z_{k} \rightarrow z_{0} \in E, k \in \Lambda$. В силу (4.1) получаем, что $f\left(z_{0}\right)=a$ и $\rho\left(z_{0}, A\right)>0$, что противоречит определению множества $A$. Таким образом, для любого $\delta>0$ и $a \in K$ существует $\varepsilon_{a}(\delta)$ такое, что $r\left(\varepsilon_{a}, a\right)<\delta$.

Перейдем к доказательству равномерной сходимости (2). Для любого $a \in K$ при фиксированном $\delta$ рассмотрим окрестность в евклидовой метрике $U_{\varepsilon(\delta) / 2}(a)$. Пусть $t \in U_{\varepsilon(\delta) / 2}(0)$. В силу того, что $|f(z)-a| \leqslant|f(z)-a-t|+|t|$, имеем

$$
\left\{z:|f(z)-a-t|<\frac{\varepsilon_{a}}{2}, z \in E\right\} \subseteq\left\{z:|f(z)-a|<\varepsilon_{a}, z \in E\right\} .
$$

Следовательно,

$$
r\left(\frac{\varepsilon_{a}(\delta)}{2}, a+t\right) \leqslant r\left(\varepsilon_{a}(\delta), a\right)<\delta, \quad t \in U_{\varepsilon_{a}(\delta) / 2}(0) .
$$

Так как $K$ - компакт, выберем конечное покрытие $K$ такими окрестностями. Взяв минимум по радиусам окрестностей из конечного покрытия, получим $\varepsilon=\varepsilon(\delta)>0$ такое, что $r(\varepsilon, a)<\delta$ для любого $a \in K$. Лемма доказана.

4.2. Перейдем к доказательству теоремы. Пусть далее $n \in \Lambda^{\varepsilon}$. Положим последовательность $\left\{\varepsilon_{n}\right\}$ из теоремы равной $1 / n$. Обозначим

$$
D_{n}:=D^{\varepsilon} \backslash K_{n}^{1 / n}
$$

и покажем, что

$$
\left[f_{n}(z)-f(z)\right] I_{D_{n}} \rightrightarrows 0,
$$

где $n \in \Lambda^{\varepsilon}$ и через $I_{M}$ обозначен индикатор множества $M$.

Из определения функции остатка (1.2) и явного вида функции $f(1.4)$ следует

$$
f_{n}(z)-f(z)=-\frac{w(z)}{q(z)} \frac{2 R_{n}\left(z^{(1)}\right)}{R_{n}\left(z^{(1)}\right)-R_{n}\left(z^{(2)}\right)} .
$$


Пусть $T_{n}(z):=R_{n}\left(z^{(2)}\right) / R_{n}\left(z^{(1)}\right)$. Тогда

$$
f_{n}(z)-f(z)=-\frac{w(z)}{q(z)} \frac{2}{1-T_{n}(z)} .
$$

Из (3.9) имеем

$$
\begin{aligned}
& T_{n}(z)=\Phi^{2(n+1-m)}(z) \exp \left\{\Omega\left(\mathbf{z}_{n}, \infty^{(2)} ; z^{(2)}\right)-\Omega\left(\mathbf{z}_{n}, \infty^{(2)} ; z^{(1)}\right)\right\} \\
& \times \exp \left\{\sum_{j=1}^{m} \Omega\left(\infty^{(1)}, a_{j}^{(2)} ; z^{(2)}\right)-\sum_{j=1}^{m} \Omega\left(\infty^{(1)}, a_{j}^{(2)} ; z^{(1)}\right)\right\} \\
& \times \exp \left\{2 \pi i \gamma_{n}\left(\Omega_{1}\left(z^{(2)}\right)-\Omega_{1}\left(z^{(1)}\right)\right)\right\} .
\end{aligned}
$$

Отсюда следует, что если $\mathbf{z}_{n} \in D^{(1)}$, то аппроксимации интерполируют искомую функцию $f, f_{n}\left(z_{n}\right)=f\left(z_{n}\right)$. Если же $\mathbf{z}_{n} \in D^{(2)}$, то получаются точки ложной интерполяции $f_{n}\left(z_{n}\right)=\widetilde{f}\left(z_{n}\right)$. Таким образом, при $n \in \Lambda^{\varepsilon}$ имеем $\mathbf{z}_{n} \in D^{(2)}$ и $\beta_{n}=z_{n}$.

Докажем, что правая часть (4.3), помноженная на $I_{D_{n}}$, равномерно стремится к бесконечности при $n \rightarrow \infty, n \in \Lambda^{\varepsilon}$. Рассмотрим $n$ такое, что $z$ принадлежит $D_{n}$. Множитель $\Phi^{2(n+1-m)}(z)$ геометрически и равномерно в области $D$ сходится к бесконечности. Два последних множителя, очевидно, равномерно по $n$ и $z$ отделены от нуля. Рассмотрим подробно выражение из первого множителя

$$
L_{n}(z):=\Omega\left(\mathbf{z}_{n}, \infty^{(2)} ; z^{(2)}\right)-\Omega\left(\mathbf{z}_{n}, \infty^{(2)} ; z^{(1)}\right) .
$$

Имеем из явного представления для абелевых дифференциалов (2.1)

$$
L_{n}(z)=-\int_{e_{1}}^{z} \frac{w\left(\mathbf{z}_{n}\right)}{w(\zeta)\left(\zeta-z_{n}\right)} d \zeta+\int_{e_{1}}^{z} \frac{\zeta-c_{n}}{w(\zeta)} d \zeta
$$

где константа $c_{n}$ обеспечивает $a$-нормированность дифференциала. Из условия $a$-нормированности $\oint_{\mathbf{a}_{1}} L_{n}(z)=0$ найдем ее явный вид:

$$
c_{n}=\left[-\frac{w\left(\mathbf{z}_{n}\right)}{z_{n}} \oint_{\mathbf{a}_{1}} \frac{d \zeta}{w(\zeta)\left(\zeta / z_{n}-1\right)}+\oint_{\mathbf{a}_{1}} \frac{\zeta}{w(\zeta)} d \zeta\right] / \oint_{\mathbf{a}_{1}} \frac{\zeta}{w(\zeta)} d \zeta .
$$

Рассмотрим случай

$$
\left|z_{n}\right| \leqslant C, \quad C \in(0, \infty), \quad \text { и } \quad\left|z-z_{n}\right|>\frac{1}{n}, \quad z \in D^{\varepsilon} .
$$

Первый интеграл в (4.4) оценивается сверху через $K_{1} \log n$, где $K_{1}$ - некая постоянная. Второй интеграл состоит из двух слагаемых. То, что включает в себя константу $c_{n}$, ограничено сверху по $z$ и с учетом (4.5) ограничено сверху по $n$. Другое слагаемое от $n$ не зависит, ведет себя в бесконечности как $\log |z|$, оставаясь ограниченным при остальных значениях $z$. Из этого анализа следует, что функция $\left|\exp \left\{L_{n}(z)\right\}\right|$ оценивается снизу как $\left|\exp \left\{L_{n}(z)\right\}\right|>K_{2} / n$, где $K_{2}$ - некая постоянная. В сумме с геометрическим возрастанием первого множителя из (4.3) получаем сходимость к бесконечности функции $T_{n}(z)$.

Теперь рассмотрим случай, когда

$$
\left|z_{n}\right|>C \text {. }
$$


Подставляя (4.5) в (4.4), получаем

$$
\begin{aligned}
L_{n}(z)=- & \frac{w\left(\mathbf{z}_{n}\right)}{z_{n}} \int_{e_{1}}^{z} \frac{d \zeta}{w(\zeta)\left(\zeta / z_{n}-1\right)}+\int_{e_{1}}^{z} \frac{\zeta}{w(\zeta)} d \zeta \\
& -\int_{e_{1}}^{z} \frac{d \zeta}{w(\zeta)}\left[-\frac{w\left(\mathbf{z}_{n}\right)}{z_{n}} \oint_{\mathbf{a}_{1}} \frac{d \zeta}{w(\zeta)\left(\zeta / z_{n}-1\right)}+\oint_{\mathbf{a}_{1}} \frac{\zeta}{w(\zeta)} d z\right] / \oint_{\mathbf{a}_{1}} \frac{d \zeta}{w(\zeta)} .
\end{aligned}
$$

Отсюда

$$
\begin{gathered}
L_{n}(z) \oint_{\mathbf{a}_{1}} \frac{d \zeta}{w(\zeta)}=\frac{w\left(\mathbf{z}_{n}\right)}{z_{n}}\left[-\int_{e_{1}}^{z} \frac{d \zeta}{w(\zeta)\left(\zeta / z_{n}-1\right)} \oint_{\mathbf{a}_{1}}^{z} \frac{d \zeta}{w(\zeta)}+\int_{e_{1}}^{z} \frac{d \zeta}{w(\zeta)} \oint_{\mathbf{a}_{1}} \frac{d \zeta}{w(\zeta)\left(\zeta / z_{n}-1\right)}\right] \\
+\int_{e_{1}}^{z} \frac{\zeta}{w(\zeta)} d \zeta \oint_{\mathbf{a}_{1}} \frac{d z}{w(z)}-\oint_{\mathbf{a}_{1}} \frac{\zeta}{w(\zeta)} d \zeta \int_{e_{1}}^{z} \frac{d \zeta}{w(\zeta)}
\end{gathered}
$$

В правой части от $n$ зависит лишь выражение в квадратных скобках и множитель перед ним. Выражение в квадратных скобках есть $O\left(1 / z_{n}\right)$ равномерно по $z \in D^{\varepsilon}$ и при $z_{n} \rightarrow \infty$. С учетом $w\left(\mathbf{z}_{n}\right) / z_{n}=O\left(z_{n}\right), z_{n} \rightarrow \infty$, и при $\rho\left(z, z_{n}\right)>1 / n$ получаем аналогично предыдущему случаю, что функция $\left|\exp \left\{L_{n}(z)\right\}\right|$ оценивается снизу как $\left|\exp \left\{L_{n}(z)\right\}\right|>K_{3} / n$, где $K_{3}-$ некая постоянная.

Объединяя рассуждения при $\left|z_{n}\right| \leqslant C$ и $\left|z_{n}\right|>C$, получаем требуемую равномерную сходимость $T_{n}(z) I_{D_{n}} \rightrightarrows 0, n \in \Lambda^{\varepsilon}$. Следовательно, получаем

$$
\left[f_{n}(z)-f(z)\right] I_{D_{n}} \rightrightarrows 0, \quad \text { где } \quad n \in \Lambda^{\varepsilon} .
$$

4.3. Докажем, что при $n \in \Lambda^{\varepsilon}$ в круге $K_{n}^{1 / n}$ содержится полюс $f_{n}$. Предположим противное. Из (4.2) следует

$$
q(z) f_{n}(z)-q(z) f(z)=-w(z) \frac{2}{1-T_{n}(z)}
$$

откуда с учетом интерполяции функции $\tilde{f}$ в точке $\beta_{n}$ и голоморфности $f_{n}$ в $K_{n}^{1 / n}$ имеем

$$
\int_{\partial K_{n}^{1 / n}} \frac{q_{n}(t) f_{n}(t)-q(t) f(t)}{t-\beta_{n}} d t=q\left(\beta_{n}\right) \tilde{f}\left(\beta_{n}\right)-q\left(\beta_{n}\right) f\left(\beta_{n}\right)=-2 w\left(\beta_{n}\right) .
$$

В силу доказанного в п. 4.2 и (4.6) левая часть (4.7) сходится к нулю, в то время как правая часть отделена от нуля. Получаем противоречие.

Докажем теперь, что при некоторой убывающей к нулю последовательности $\left\{\rho_{n}\right\}$ круг $K_{n}^{\rho_{n}}$, начиная с некоторого $n$, содержит произвольную $A$-точку функции $f_{n}$, $A \in \mathbb{C}$. Воспользуемся принципом аргумента. Пусть $l_{n}-$ произвольный замкнутый контур, содержащий круг $K_{n}^{\rho_{n}}$ и содержащийся в области $D^{\varepsilon}$. Имеем вдоль $l_{n}$ :

$$
\frac{1}{2 \pi} \Delta \arg \left[f_{n}(z)-A\right]=\frac{1}{2 \pi} \Delta \arg [f(z)-A]+\frac{1}{2 \pi} \Delta \arg \left[1+\frac{f_{n}(z)-f(z)}{f(z)-A}\right] .
$$

В силу голоморфности функции $f$ в $D^{\varepsilon}$ первое слагаемое в правой части (4.8) равно количеству $A$-точек функции $f$ во внутренности $l_{n}$ и, следовательно, неотрицательно. Для доказательства теоремы осталось показать, что для каждого $n$ существует контур $l_{n}$, при котором второе слагаемое, начиная с некоторого $n$, равномерно по 
всем $A \in \overline{\mathbb{C}}$ равно нулю. Действительно, в этом случае левая часть (4.8) неотрицательна. Так как $f_{n}$ имеет внутри $l_{n}$ по крайней мере один полюс, то получаем, что там же содержится по крайней мере одна $A$-точка аппроксимации.

Для доказательства равенства

$$
\frac{1}{2 \pi} \Delta \arg \left[1+\frac{f_{n}(z)-f(z)}{f(z)-A}\right]=0
$$

на некотором контуре $l_{n}$ достаточно показать, что на том же контуре выполняется неравенство

$$
\left|f_{n}(z)-f(z)\right|<|f(z)-A| .
$$

Функция $f$ ограничена в $D^{\varepsilon},|f|<M$. Пусть $|A|>2 M$. Тогда $|f-A|$ равномерно ограничено снизу, а $\left|f_{n}(z)-f(z)\right|$ - в силу результатов раздела 2 , равномерно на контуре $l_{n}$ сходится к нулю. Получаем (4.10).

Пусть $|A| \leqslant 2 M$. Тогда $A$ принадлежит компакту и можно воспользоваться леммой из раздела 1 . Из нее следует, что множество

$$
\left\{z:|f(z)-A| \leqslant\left|f_{n}(z)-f(z)\right|\right\}, \quad \text { где } \quad\left|f_{n}(z)-f(z)\right| \rightarrow 0, \quad n \in \Lambda^{\varepsilon},
$$

содержится в ограниченном числе кругов со сходящимися к нулю радиусами $r_{n}$. Если число кругов оценить сверху константой $s$ и взять в качестве $\rho_{n}$ в доказываемой теореме $s r_{n}$, получим существование необходимого контура $l_{n}$ в $K_{n}^{\rho_{n}}$. Теорема доказана.

В заключение автор выражает благодарность С. П. Суетину за постановку задачи и внимание к работе, а В.И. Буслаеву за замечания, способствующие улучшению текста работы.

\section{СПИСОК ЦИТИРОВАННОЙ ЛИТЕРАТУРЫ}

[1] H. Stahl, "The convergence of Padé approximants to functions with branch points", J. Approx. Theory, 91:2, (1997), 139-204.

[2] H. Stahl, "Diagonal Padé approximants to hyperelliptic functions", Ann. Fac. Sci. Toulouse Math. (6), 1996, Special issue, 121-193.

[3] H. Stahl, "Spurious poles in Padé approximation", J. Comput. Appl. Math., 99:1-2, (1998), 511-527.

[4] С.П. Суетин, "О сходимости чебышёвских непрерывных дробей для эллиптических функций”, Матем. сб., 194:12, (2003), 63-92.

[5] С.П. Суетин, "О равномерной сходимости диагональных аппроксимаций Паде для гиперэллиптических функций”, Матем. сб., 191:9, (2000), 81-114.

[6] С. П. Суетин, "Об интерполяционных свойствах диагональных аппроксимаций Паде эллиптических функций", УМН, 59:4, (2004), 201-202.

[7] Дж. Бейкер мл., П. Грейвс-Моррис, Аппроксимации Паде. Ч. 1. Основы теории. Ч. 2. Обобщения и приложения, Мир, М., 1986.

[8] J. Gammel, W. Marshall, L. Morgan, "An application of Padé approximants to Heisenberg ferromagnetism and antiferromagnetism", Proc. R. Soc. Lond. Ser. A Math. Phys. Eng. Sci., 275:1361, (1963), 257-270.

[9] В. А. Ильина, П. К. Силаев, Численные методъ для физиков-теоретиков, Ч. 1, ИКИ, М, Ижевск, 2003.

[10] S. Dumas, Sur le développement des fonctions elliptiques en fractions continues, Thesis, Zürich, 1908, 59 pp. 
[11] В. И. Буслаев, "О гипотезе Бейкера-Гаммеля-У иллса в теории аппроксимаций Паде", Матем. сб., 193:6, (2002), 25-38.

[12] Д. В. Христофоров, "О сходимости диагональных аппроксимаций Паде для эллиптических функций", Матем. сб., 200:6, (2009), 143-160.

[13] Э. И. Зверович, "Краевые задачи теории аналитических функций в гёльдеровских классах на римановых поверхностях", УМН, 26:1, (1971), 113-179.

[14] О. Форстер, Римановы поверхности, Мир, М., 1980.

Д. В. Христофоров

Поступило

Математический институт им. В. А. Стеклова РАН

31.03.2009

E-mail: khrden@gmail.com

Исправленный вариант

29.10.2009 